\title{
Effect of I/Q Imbalance on Pilot Design for MIMO OFDM Channel Estimation
}

\author{
Hlaing Minn and Daniel Munoz \\ Department of Electrical Engineering, University of Texas at Dallas \\ Email: \{hlaing.minn,djm072000\}@utdallas.edu
}

\begin{abstract}
Efficient pilot designs are vital as they improve system performance and resource utilization, especially in multiinput multi-output (MIMO) orthogonal frequency divison multiplexing (OFDM) systems. Most of the existing pilot designs do not consider in-phase and quadrature (I/Q) imbalance whose detrimental effects become more severe since the technology and applications are pushing towards smaller semiconductor node, smaller chip, cheaper equipment, and larger modulation order. This paper studies the impact of $I / Q$ imbalance on the pilot designs, and presents several new pilot designs for equivalent channel estimation of MIMO OFDM systems with frequencydependent I/Q imbalances. Advantages of the proposed designs are illustrated through simulation and analytical results in terms of estimation accuracy, error rate, overhead efficiency, and generality with the transmission formats (preamble-based or pilot-data-multiplexed systems, with or without null guard tones).
\end{abstract}

\section{INTRODUCTION}

In channel estimation of MIMO OFDM systems, pilots are typically used. Efficient pilot designs for MIMO systems are more challenging than those for single antenna systems since interferences among multiple antennas need to be avoided. Another source of challenge that further complicates the pilot designs is in-phase and quadrature (I/Q) imbalance which represents the gain and phase imbalances across the inphase and the quadrature branches of the transmitter or/and the receiver, as well as across the frequency (frequencydependent imbalance) within the transmission band. As the semiconductor industry continues its drive toward smaller chips with the same or higher processing power and lower cost, the accuracy of these devices is continually the casualty and I/Q imbalance becomes a greater problem. In addition, different RF chains may have different I/Q imbalances which enlarges the dimension of the unknown parameters. These facts highlight a necessity to develop overhead-efficient estimationoptimal pilot designs for such systems.

Pilot designs for MIMO OFDM systems without I/Q imbalances have been considered in [1], [2] and references therein. Compensation algorithms or pilot designs for I/Q imbalance have been addressed for SISO systems in [3]-[14]). Recently, [16] proposes a pilot design for MIMO OFDM systems with $\mathrm{I} / \mathrm{Q}$ imbalance, but it is overhead-inefficient and does not consider null guard tones in the design. In practical OFDM systems, there are several null guard tones at the band edges for easier filter implementation and emission spectrum control. Hence, pilot designs should incorporate the constraint of null guard tones for practical systems.
In this paper we study the effect of I/Q imbalance on the channel estimation and pilot designs for MIMO OFDM systems. We develop several pilot designs with null guard tones in the presence of transmit and receive frequency-dependent $\mathrm{I} / \mathrm{Q}$ imbalances. The proposed designs provide estimation performance optimality, minimal overhead, and generality in transmission format (e.g., preamble or pilot-data multiplexed setup, with null guard tones, etc.), hence overcoming the limitations and disadvantages of the existing methods.

Notation: A bold capital (small) letter represents a matrix (a column vector). The superscripts $*, T$, and $H$ represent the conjugate, the transpose, and the conjugate transpose operations, respectively. $[\boldsymbol{X}]_{k, m}$ denotes the $k$-th row, $m$-th column element of $\boldsymbol{X}$. All indices start from 0 . The all-zero square matrix of size- $k$, the $k \times m$ all-zero matrix, and the $k \times k$ identity matrix are denoted by $\mathbf{0}_{k}, \mathbf{0}_{k \times m}$, and $\boldsymbol{I}_{k}$, respectively. $\operatorname{Tr}[\boldsymbol{X}]$ denotes the trace of $\boldsymbol{X}$. $\operatorname{diag}\{\boldsymbol{x}\}$ represents a diagonal matrix whose diagonal elements are defined by $\boldsymbol{x}$. The $l$-cyclic-forward-shifted version of $c$ is denoted by $c^{(l)}$. $*$ and $\otimes$ denote the convolution and the Kronecker product, respectively. $(l)_{N}$ represents $l$ modulo $N .\lfloor X\rfloor$ denotes the integer part of $X$ while $\lceil X\rceil$ represents the smallest integer greater than or equal to $X$. The subcarrier indexes are modulo $N$ where $N$ is the total number of OFDM subcarriers. We will often use $-k$ to denote the subcarrier index $(-k)_{N}$.

\section{Signal Model}

First, consider a single antenna system with both transmit and receive I/Q imbalances where $\left\{a_{t}^{I}, a_{t}^{Q}\right\}$ and $\left\{\theta_{t}^{I}, \theta_{t}^{Q}\right\}$ represent frequency-independent gains and phase offsets of the I and $\mathrm{Q}$ branches at the transmitter. The equivalent pulse shaping filters (the overall impulse responses including DAC, amplifier, pulse shaping, and frequency-dependent I/Q imbalances) for the I and Q branches of the transmitter are denoted by $g_{t}^{I}(t)$ and $g_{t}^{Q}(t)$. The receiver side parameters are defined in the same manner with the subscript $t$ replaced by $r$. The transmit system with I/Q imbalance can be viewed as the summation of two systems namely direct system whose input is the same as the transmitter input signal $s(t)=s_{I}(t)+j s_{Q}(t)$ and mirror system whose input is $s^{*}(t)$. The impulse responses of the direct and mirror systems at the transmitter are denoted by $g_{T}^{D}(t)$ and $g_{T}^{M}(t)$, and those at the receiver are represented by 
$g_{R}^{D}(t)$ and $g_{R}^{M}(t)$, respectively. They are given as

$$
\begin{aligned}
g_{T}^{D}(t) & =\left[a_{t}^{I} e^{j \theta_{t}^{I}} g_{t}^{I}(t)+a_{t}^{Q} e^{j \theta_{t}^{Q}} g_{t}^{Q}(t)\right] / 2 \\
g_{T}^{M}(t) & =\left[a_{t}^{I} e^{j \theta_{t}^{I}} g_{t}^{I}(t)-a_{t}^{Q} e^{j \theta_{t}^{Q}} g_{t}^{Q}(t)\right] / 2 \\
g_{R}^{D}(t) & =\left[a_{r}^{I} e^{-j \theta_{r}^{I}} g_{r}^{I}(t)+a_{r}^{Q} e^{-j \theta_{r}^{Q}} g_{r}^{Q}(t)\right] / 2 \\
g_{R}^{M}(t) & =\left[a_{r}^{I} e^{j \theta_{r}^{I}} g_{r}^{I}(t)-a_{r}^{Q} e^{j \theta_{r}^{Q}} g_{r}^{Q}(t)\right] / 2 .
\end{aligned}
$$

The above signal model can be extended to MIMO systems where different RF chains corresponding to different antennas may have different I/Q imbalances. The above impulse responses corresponding to the $i$ th transmit antenna and the $j$ th receive antenna are denoted by $g_{T, i}^{D}(t), g_{T, i}^{M}(t), g_{R, j}^{D}(t)$, and $g_{R, j}^{M}(t)$, respectively. A simplified low-pass-equivalent signal model for MIMO is presented in Fig. 1 for the $j$ th receive antenna where the impulse responses of the overall direct channel $p_{i, j}(t)$ and the overall mirror channel $q_{i j}(t)$ corresponding to the $i$ th transmit antenna and the $j$ th receive antenna are given by

$$
\begin{aligned}
p_{i j}(t) & =g_{T, i}^{D}(t) * h_{i j}(t) * g_{R, j}^{D}(t) \\
& +\left(g_{T, i}^{M}(t)\right)^{*} * h_{i j}^{*}(t) * g_{R, j}^{M}(t) \\
q_{i j}(t) & =g_{T, i}^{M}(t) * h_{i j}(t) * g_{R, j}^{D}(t) \\
& +\left(g_{T, i}^{D}(t)\right)^{*} * h_{i j}^{*}(t) * g_{R, j}^{M}(t) .
\end{aligned}
$$

The receive filter output signal corresponding to the receive antenna $j$ and transmit signals $\left\{s_{i}(t)\right\}$ is

$$
r_{j}(t)=\sum_{i=0}^{N_{\mathrm{Tx}}-1}\left(s_{i}(t) * p_{i}(t)+s_{i}^{*}(t) * q_{i}(t)\right)+n_{j}(t)
$$

where the complex Gaussian noise $n_{j}(t)$ is given by

$$
n_{j}(t)=w(t) * g_{R, j}^{D}(t)+w^{*}(t) * g_{R, j}^{M}(t) .
$$

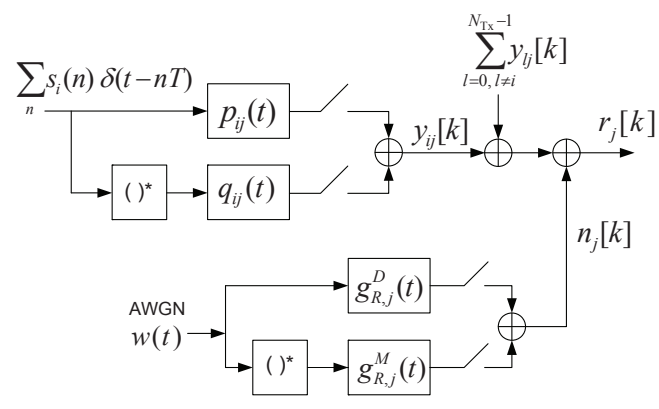

Fig. 1. A simplified equivalent lowpass MIMO system model with frequencydependent I/Q imbalances

When MIMO channels are independent or their joint statistics are unknown, the logical approach to the estimation of the equivalent direct and mirror channels is to estimate them at each receive antenna independently. Hence, we just need to describe them for one receive antenna. In the following we drop the receive antenna index $j$. We consider an OFDM system with a cyclic prefix $(\mathrm{CP})$ interval $\left(N_{\mathrm{CP}}\right.$ samples) longer than the maximum span ( $L$ samples) of $\left\{p_{i}(t)\right\}$ and $\left\{q_{i}(t)\right\}$.

Now, let us consider a low-pass-equivalent discrete-time OFDM system with $N$ subcarriers. The channels are assumed to be constant over $K$ symbol intervals. The discrete-time transmit training signal from the $i$ th transmit antenna during the $l$ th symbol duration is denoted by $s_{i}^{(l)}[k]$ with the integer index $k \in\left[-N_{\mathrm{CP}}, N-1\right]$, and the CP samples $s_{i}^{(l)}[m]=$ $s_{i}^{(l)}[N-m]$ for $m \in\left[-N_{\mathrm{CP}},-1\right]$. Similar notations apply to data signal $x_{i}^{(l)}[k]$. The discrete-time versions of $p_{i}(t)$ and $q_{i}(t)$ are denoted by $L \times 1$ vectors $\boldsymbol{p}_{i}$ and $\boldsymbol{q}_{i}$, respectively. The time-domain $N \times 1$ received signal vector after $\mathrm{CP}$ removal at each receive antenna for the $l$ th OFDM symbol is denoted by $r_{l}$ which can be expressed in a general pilot-data multiplexed setup (which includes pilot only or data only symbols as special cases) as

$$
\boldsymbol{r}_{l}=\sum_{i=0}^{N_{\mathrm{Tx}}-1}\left\{\left(\boldsymbol{S}_{i}[l]+\boldsymbol{X}_{i}[l]\right) \boldsymbol{p}_{i}+\left(\boldsymbol{S}_{i}^{*}[l]+\boldsymbol{X}_{i}^{*}[l]\right) \boldsymbol{q}_{i}\right\}+\boldsymbol{n}_{l}
$$

where $(m, k)$ th element of the $N \times L$ signal matrix $\boldsymbol{S}_{i}[l]$ (or $\left.\boldsymbol{X}_{i}[l]\right)$ is $s_{i}^{(l)}[m-k]$ (or $x_{i}^{(l)}[m-k]$ ) with $m \in[0, N-1]$ and $k \in[0, L-1]$. The received signal vector for $K$ OFDM symbols is given by

$$
\boldsymbol{r}=\boldsymbol{S p}+\boldsymbol{S}^{*} \boldsymbol{q}+\boldsymbol{X} \boldsymbol{p}+\boldsymbol{X}^{*} \boldsymbol{q}+\boldsymbol{n}
$$

where $\boldsymbol{r}=\left[\boldsymbol{r}_{0}^{T} \boldsymbol{r}_{1}^{T} \ldots \boldsymbol{r}_{K-1}^{T}\right]^{T}, \boldsymbol{p}=\left[\boldsymbol{p}_{0}^{T} \boldsymbol{p}_{1}^{T} \ldots \boldsymbol{p}_{N_{\mathrm{Tx}-1}}^{T}\right]^{T}, \boldsymbol{q}=$ $\left[\boldsymbol{q}_{0}^{T} \boldsymbol{q}_{1}^{T} \ldots \boldsymbol{q}_{N_{\text {Tx }-1}}^{T}\right]^{T}, \boldsymbol{n}=\left[\boldsymbol{n}_{0}^{T} \boldsymbol{n}_{1}^{T} \ldots \boldsymbol{n}_{K-1}^{T}\right]^{T}$, the $(k, i)$ th submatrice of $\boldsymbol{S}$ and $\boldsymbol{X}$ are respectively given by $\boldsymbol{S}_{i}[k]$ and $\boldsymbol{X}_{i}[k]$, with $k \in[0, K-1]$ and $i \in\left[0, N_{\mathrm{Tx}}-1\right]$. The complex Gaussian noise vectors $\left\{\boldsymbol{n}_{l}\right\}$ are given by

$$
\boldsymbol{n}_{l}=\boldsymbol{G}_{R, D} \boldsymbol{w}_{l}+\boldsymbol{G}_{R, M} \boldsymbol{w}_{l}^{*}
$$

where $\left\{\boldsymbol{w}_{l}\right\}$ are independent and identically distributed (iid) random vectors, each consisting of iid circularly-symmetric complex Gaussian random variables with the variance $\sigma_{w}^{2}$. Let $\lambda$ denote the maximum of the number of taps of $g_{R}^{D}[k]$ and $g_{R}^{M}[k]$. Then, $\boldsymbol{G}_{R, D}$ and $\boldsymbol{G}_{R, M}$ are $N \times(N+\lambda)$ matrices with their first rows given by $\left[g_{R}^{D}[0], g_{R}^{D}[1], \ldots, g_{R}^{D}[\lambda], \mathbf{0}_{1 \times N-1}\right]$ and $\left[g_{R}^{M}[0], g_{R}^{M}[1], \ldots, g_{R}^{M}[\lambda], \mathbf{0}_{1 \times N-1}\right]$, respectively, and their next $k$ th rows are cyclically $k$-right-shift versions of their corresponding first rows.

\section{MIMO OFDM PILOT DESIGN CRITERIA}

For coherent detection, the direct channel $\boldsymbol{p}$ and the mirror channel $\boldsymbol{q}$ need to be estimated at the receiver. In practical systems, the statistics of the channel and the transceiver imperfections are unknown and they can be non-stationary as well. This leads to the practical choice of least-squares type channel estimators as considered in this paper. The estimates of the direct and mirror CIR vectors are given by

$$
\begin{aligned}
& \hat{\boldsymbol{p}}=\left(\boldsymbol{S}^{H} \boldsymbol{S}\right)^{-1} \boldsymbol{S}^{H} \boldsymbol{r} \\
& \hat{\boldsymbol{q}}=\left(\boldsymbol{S}^{T} \boldsymbol{S}^{*}\right)^{-1} \boldsymbol{S}^{T} \boldsymbol{r} .
\end{aligned}
$$

Our pilot designs will be based on minimizing the mean-square error (MSE) of the channel estimation.

We observe the following conditions for the pilot designs: 
1) Estimation Identifiability Condition: The identifiability of $\boldsymbol{p}$ and $\boldsymbol{q}$ estimation requires that $\boldsymbol{S}^{H} \boldsymbol{S}$ is of full-rank.

2) Zero Cross Channel Interference Condition: The interference from the mirror channel is removed when $\boldsymbol{S}^{H} \boldsymbol{S}^{*}=\mathbf{0}_{L N_{\mathrm{Tx}}}$.

3) Zero Data Interference Condition: The random data interference is completely suppressed when $\boldsymbol{S}^{H} \boldsymbol{X}=$ $\mathbf{0}_{L N_{\mathrm{Tx}}}$ and $\boldsymbol{S}^{H} \boldsymbol{X}^{*}=\mathbf{0}_{L N_{\mathrm{Tx}}}$.

4) White Noise Optimality Condition: For the scenario where the equivalent receive-filter is a square-root Nyquist filter, the MSE due to the noise is minimized when $\boldsymbol{S}^{H} \boldsymbol{S}=E_{K} \boldsymbol{I}_{L N_{\mathrm{Tx}}}$ where $E_{K}$ is the total energy of the transmit signal vector from a transmit antenna over $K$ symbols (excluding CPs). The frequencyindependent receiver I/Q imbalance with a square-root raised cosine receive filter represents this scenario.

5) For the scenario with frequency-dependent receiver I/Q imbalance, the noise covariance matrix is unknown a priori, and hence it is infeasible to develop optimal pilot designs. However, frequency selectivity of the receiver I/Q imbalance is typically very small. A practical approach in this case is to assume frequency-flat receiver I/Q imbalance in the pilot designs which leads to the requirement of $\boldsymbol{S}^{H} \boldsymbol{S}=E_{K} \boldsymbol{I}_{L N_{\mathrm{Tx}}}$.

When the identifiability condition and the zero data interference condition are satisfied, the MSEs of $\boldsymbol{p}$ and $\boldsymbol{q}$ estimation are given by

$$
\begin{aligned}
& \operatorname{MSE}_{\boldsymbol{p}}=\operatorname{Tr} {\left[\left(\boldsymbol{S}^{H} \boldsymbol{S}\right)^{-1} \boldsymbol{S}^{H} \boldsymbol{S}^{*} E\left[\boldsymbol{q} \boldsymbol{q}^{H}\right] \boldsymbol{S}^{T} \boldsymbol{S}\left(\boldsymbol{S}^{H} \boldsymbol{S}\right)^{-1}\right.} \\
&+\left.\left(\boldsymbol{S}^{H} \boldsymbol{S}\right)^{-1} \boldsymbol{S}^{H} \boldsymbol{C}_{\boldsymbol{n}} \boldsymbol{S}\left(\boldsymbol{S}^{H} \boldsymbol{S}\right)^{-1}\right] \\
& \mathrm{MSE}_{\boldsymbol{q}}=\operatorname{Tr}\left[\left(\boldsymbol{S}^{T} \boldsymbol{S}^{*}\right)^{-1} \boldsymbol{S}^{T} \boldsymbol{S} E\left[\boldsymbol{p} \boldsymbol{p}^{H}\right] \boldsymbol{S}^{H} \boldsymbol{S}^{*}\left(\boldsymbol{S}^{T} \boldsymbol{S}^{*}\right)^{-1}\right. \\
&\left.+\left(\boldsymbol{S}^{T} \boldsymbol{S}^{*}\right)^{-1} \boldsymbol{S}^{T} \boldsymbol{C}_{\boldsymbol{n}} \boldsymbol{S}^{*}\left(\boldsymbol{S}^{T} \boldsymbol{S}^{*}\right)^{-1}\right]
\end{aligned}
$$

where the noise covariance matrix $C_{n}$ is given by

$$
\boldsymbol{C}_{\boldsymbol{n}}=\sigma_{w}^{2}\left(\boldsymbol{G}_{R, D} \boldsymbol{G}_{R, D}^{H}+\boldsymbol{G}_{R, M} \boldsymbol{G}_{R, M}^{H}\right) \otimes \boldsymbol{I}_{K} .
$$

If the zero cross channel interference condition is also met, the above MSE expressions become

$$
\begin{aligned}
& \mathrm{MSE}_{\boldsymbol{p}}=\operatorname{Tr}\left[\left(\boldsymbol{S}^{H} \boldsymbol{S}\right)^{-1} \boldsymbol{S}^{H} \boldsymbol{C}_{\boldsymbol{n}} \boldsymbol{S}\left(\boldsymbol{S}^{H} \boldsymbol{S}\right)^{-1}\right] \\
& \mathrm{MSE}_{\boldsymbol{q}}=\operatorname{Tr}\left[\left(\boldsymbol{S}^{T} \boldsymbol{S}^{*}\right)^{-1} \boldsymbol{S}^{T} \boldsymbol{C}_{\boldsymbol{n}} \boldsymbol{S}^{*}\left(\boldsymbol{S}^{T} \boldsymbol{S}^{*}\right)^{-1}\right] .
\end{aligned}
$$

Additionally, if the demodulator output noise samples are white (i.e., $\boldsymbol{C}_{\boldsymbol{n}}=\sigma_{n}^{2} \boldsymbol{I}$ ), the MSE expressions simplify to $\operatorname{MSE}_{\boldsymbol{p}}=\mathrm{MSE}_{\boldsymbol{q}}=\sigma_{n}^{2} \operatorname{Tr}\left[\left(\boldsymbol{S}^{H} \boldsymbol{S}\right)^{-1}\right]$.

The MIMO OFDM pilot design criterion satisfying estimation identifiability, zero data interference condition, zero cross channel interference, and white noise optimality reads as

$$
\left.\begin{array}{l}
\left(\boldsymbol{S}^{H} \boldsymbol{S}\right)=E_{K} \boldsymbol{I}_{N_{\mathrm{Tx}} L} \quad \& \quad\left(\boldsymbol{S}^{H} \boldsymbol{S}^{*}\right)=\mathbf{0}_{N_{\mathrm{Tx}} L} \\
\boldsymbol{S}^{H} \boldsymbol{X}=\mathbf{0}_{N_{\mathrm{Tx}} L} \quad \& \quad \boldsymbol{S}^{H} \boldsymbol{X}^{*}=\mathbf{0}_{N_{\mathrm{Tx}} L}
\end{array}\right\}
$$

and the pilot tones cannot overlap with the guard band. The corresponding MSE becomes

$$
\begin{aligned}
\mathrm{MSE}_{\boldsymbol{p}}=\mathrm{MSE}_{\boldsymbol{q}} & =\frac{\sigma_{w}^{2}}{E_{K}^{2}} \operatorname{Tr}\left[\left(\boldsymbol { S } ^ { H } \left(\left(\boldsymbol{G}_{R, D} \boldsymbol{G}_{R, D}^{H}\right.\right.\right.\right. \\
& \left.\left.\left.\left.+\boldsymbol{G}_{R, M} \boldsymbol{G}_{R, M}^{H}\right) \otimes \boldsymbol{I}_{K}\right) \boldsymbol{S}\right)^{-1}\right] .
\end{aligned}
$$

\section{PRoposed MIMO OFDM PILOT DESIGNS}

Let $c_{i, l}[k]$ and $b_{i, l}[k]$, respectively, denote the pilot symbol and the data symbol on the $k$ th subcarrier of the $l$ th OFDM symbol from the $i$ th transmit antenna. Define $\mathcal{J}_{l} \triangleq$ $\cup_{i=0}^{N_{\mathrm{Tx}}-1} \mathcal{J}_{l, i}$ and $\mathcal{I}_{l} \triangleq \cup_{i=0}^{N_{\mathrm{Tx}}-1} \mathcal{I}_{l, i}$ where $\mathcal{J}_{l, i}$ and $\mathcal{I}_{l, i}$ denote the pilot (including null pilot) index set and the data index set of $i$ th antenna at $l$ th OFDM symbol, respectively. Note that $\mathcal{J}_{l, i}$ can be separated into non-zero pilot tone index set $\mathcal{J}_{l, i}^{\text {pilot }}$ and null pilot tone index set $\mathcal{J}_{l, i}^{\text {null }}$. Also define $L_{0} \triangleq 2^{\left\lceil\log _{2}(L)\right\rceil}$, $L_{i}=2^{i} L_{0}$, and $M_{i}=N / L_{i}$.

The proposed designs will be denoted by two terms - how pilots of different antennas are decoupled and how mirror tone interferences are suppressed. The same pilot signal energy over $K$ symbols for each antenna as required in Condition3 will not be explicitly mentioned in the following designs. Without loss of generality, we will present unit-amplitude pilot symbols, as any common amplitude scaling does not affect pilot designs. The existing MIMO OFDM pilot designs from [2], namely code division multiplexing across time domain (CDM-T), code division multiplexing across frequency domain (CDM-F), and frequency division multiplexing (FDM), will be extensively used in our designs. Due to space limitation, details of these existing designs are referred to [2]. How our pilot designs satisfy the five design criteria will be briefly mentioned for the first design, but it should be obvious and hence will be skipped for the other designs. The proposed designs can work in the system with a maximum of $M_{0}-2 N_{\mathrm{Tx}}-1$ total null guard tones for $N_{\mathrm{Tx}}>1$.

\section{A. [CDM-F; Null] Design}

This design uses $V L_{m}$ constant amplitude pilot tones with the index set $\mathcal{J}_{0, i}^{\text {pilot }}=\mathcal{J}_{0}^{\text {pilot }}$ and $V L_{m}$ null tones with the index set $\mathcal{J}_{0, i}^{\text {null }}=\mathcal{J}_{0}^{\text {null }}$ where $\mathcal{J}_{0}^{\text {pilot }}$ and $\mathcal{J}_{0}^{\text {null }}$ are mirrors of each other, and $V \geq N_{\mathrm{Tx}}$ and $V L_{m} \leq N / 2$. Self-mirror tones cannot be used. Define $\mathcal{T}_{n, k} \triangleq\left[k, k+M_{n}, k+2 M_{n}\right.$, $\left.\ldots, k+N-M_{n}\right]$ which consists of cyclically equi-spaced $L_{n}$ indexes from $[0, N-1]$. Then the index sets are given by

$$
\begin{aligned}
\mathcal{J}_{0, i}^{\text {pilot }} & =\mathcal{J}_{0}^{\text {pilot }}=\bigcup_{k=0}^{V-1} \mathcal{T}_{n, \tau_{k}}, \\
\mathcal{J}_{0, i}^{\text {null }} & =\mathcal{J}_{0}^{\text {null }}=\left\{N-\mathcal{J}_{0}^{\text {pilot }}\right\} \\
\mathcal{I}_{l} & =\{0,1,2, \ldots, N-1\} \backslash\left\{\mathcal{J}_{0}^{\text {pilot }} \cup \mathcal{J}_{0}^{\text {null }}\right\}
\end{aligned}
$$

where $\tau_{k} \in\left\{\left\{1,2, \ldots, M_{n}-1\right\} \backslash\left\{M_{n} / 2\right\}\right\}, \tau_{k} \neq \tau_{n}$ if $k \neq$ $n,\left\{\tau_{k}\right\} \cap\left\{M_{n}-\tau_{k}\right\}=\emptyset$, and $M_{n} \geq 2 N_{\mathrm{Tx}}+2$.

The assignment of the pilot, null and data indices in (21), (22), and (23) guarantee that the zero data interference condition is met. Due to the mirror null tones, the I/Q imbalance 
induced interferences are completely suppressed, satisfying the zero cross channel interference condition. The pilots of different antennas are decoupled by CDM-F design as

$$
\begin{aligned}
\boldsymbol{c}_{0}[k]= & e^{j \phi_{k}}, \text { arbitrary } \phi_{k}, k \in \mathcal{J}_{0}^{\text {pilot }} \\
\boldsymbol{c}_{i}[k]= & e^{j 2 \pi m i / K} \boldsymbol{c}_{0}[k], \quad k \in \mathcal{T}_{n, \tau_{m}}, \\
& i \in\left\{1,2, \ldots, N_{\mathrm{Tx}}-1\right\} \\
\boldsymbol{c}_{i}[k]= & 0, k \notin \mathcal{J}_{0}^{\text {pilot }}, i \in\left\{0,1, \ldots, N_{\mathrm{Tx}}-1\right\} .
\end{aligned}
$$

If the elements of $\mathcal{J}_{0}^{\text {pilot }}$ are cyclically equi-spaced, the pilots can also be given by

$$
\begin{aligned}
\boldsymbol{c}_{0}[k]= & e^{j \phi_{0, k}}, k \in \mathcal{J}_{0}^{\text {pilot }} \\
\boldsymbol{c}_{i}[k]= & e^{j \phi_{i}} e^{j 2 \pi k \tau_{i} / N} \boldsymbol{c}_{0}[k], i \in\left\{1,2, \ldots, N_{\mathrm{Tx}}-1\right\} \\
& L \leq \tau_{1} \leq L_{n}, L \leq \tau_{i+1}-\tau_{i} \leq L_{n} \\
\boldsymbol{c}_{i}[k]= & 0, \quad k \notin \mathcal{J}_{0}^{\text {pilot }}, i \in\left\{0,1, \ldots, N_{\mathrm{Tx}}-1\right\}
\end{aligned}
$$

where $\left\{\phi_{i}, \phi_{0, k}\right\}$ are arbitrary phases. The CDM-F assignment of the pilots guarantee the estimation identifiability and white noise optimality conditions are met. The choice of $V=N_{\mathrm{Tx}}$ and $L_{m}=L_{0}$ requires minimum overhead.

\section{B. [FDM; Null] Design}

This design decouples pilots of different antennas through FDM design and suppresses mirror tone interferences by means of mirror null tones. It uses $K=1$ symbol with $N_{\text {Tx }} L_{m} \leq N / 2$, and consists of $N_{\mathrm{Tx}} L_{m}$ constant amplitude pilot tones with the index set $\mathcal{J}_{0}^{\text {pilot }}=\cup_{i} \mathcal{J}_{0, i}^{\text {pilot }}$ and $N_{\text {Tx }} L_{m}$ null tones with the index set $\mathcal{J}_{0}^{\text {null }}$. The differences from [CDM-F; Null] design are (i) the constant amplitude pilots can be arbitrary within and across antennas in this design while they are dependent across antennas in [CDM-F; Null] design and (ii) a different antenna transmits its constant amplitude pilots only on distinct cyclically equi-spaced $L_{m}$ tones in this design while each antenna uses the same $N_{\mathrm{Tx}} L_{m}$ tones in [CDM-F; Null] design. The index sets of [FDM; Null] design are defined by

$$
\begin{aligned}
\mathcal{J}_{0, i}^{\text {pilot }} & =\mathcal{I}_{n, \tau_{i}}, \tau_{k} \neq \tau_{n} \text { if } k \neq n, \\
\mathcal{J}_{0, i}^{\text {null }} & =\mathcal{J}_{0}^{\text {null }}=\left\{N-\mathcal{J}_{0}^{\text {pilot }}\right\} \\
\mathcal{I}_{l} & =\{0,1,2, \ldots, N-1\} \backslash\left\{\mathcal{J}_{0}^{\text {pilot }} \cup \mathcal{J}_{0}^{\text {null }}\right\}
\end{aligned}
$$

where $\tau_{k} \in\left\{\left\{1,2, \ldots, M_{n}-1\right\} \backslash\left\{M_{n} / 2\right\}\right\},\left\{\tau_{k}\right\} \cap\left\{M_{n}-\right.$ $\left.\tau_{k}\right\}=\emptyset$.

The pilot tones are given by

$$
\begin{aligned}
\boldsymbol{c}_{i}[k]= & e^{j \phi_{i, k}}, \text { arbitrary } \phi_{i, k}, \\
& k \in \mathcal{J}_{0, i}^{\text {pilot }}, i \in\left\{0,1, \ldots, N_{\mathrm{Tx}}-1\right\} \\
\boldsymbol{c}_{i}[k]= & 0, k \notin \mathcal{J}_{0, i}^{\text {pilot }} .
\end{aligned}
$$

\section{C. [CDM-F or FDM; C-T] Design}

This design uses two OFDM symbols. In CDM-F, each antenna transmits $N_{\mathrm{Tx}} L_{n}$ constant-amplitude pilot tones in each symbol, while in FDM each antenna transmits $L_{n}$ constantamplitude pilot tones and $\left(N_{\mathrm{Tx}}-1\right) L_{n}$ null pilot tones in each symbol. These pilot indexes are all mirror-pairs (may include self-mirror tones for systems without null guard tones). The index sets are given by

$$
\begin{aligned}
\mathcal{J}_{l} & =\bigcup_{m=0}^{N_{\mathrm{Tx}}-1} \mathcal{T}_{n, \tau_{m}}, \tau_{m} \in\left\{0,1, \ldots, M_{n}-1\right\}, \\
\mathcal{I}_{l} & =\{0,1,2, \ldots, N-1\} \backslash \mathcal{J}_{l}, \\
\mathcal{J}_{l, i} & = \begin{cases}\mathcal{J}_{l}, & \text { CDM-F } \\
\mathcal{T}_{n, \tau_{i}}, & \text { FDM }\end{cases}
\end{aligned}
$$

where $\tau_{m} \neq \tau_{k}$ if $m \neq k$ and $\left\{\tau_{m}\right\}=\left\{\left(M_{n}-\tau_{m}\right)_{M_{n}}\right\}$.

The choice of $n=0$ in $L_{n}$ and $M_{n}$ yields minimum pilot overhead. The pilots from different antennas are decoupled by CDM-F or FDM, while the mirror tone interferences are suppressed by C-T over two symbols. For CDM-F, each antenna transmits constant amplitude pilots on $\mathcal{J}_{l}$. For FDM, the $m$ th antenna transmits constant amplitude pilots on $\mathcal{T}_{n, \tau_{m}}$ and null tones on $\left\{\mathcal{J}_{l} \backslash \mathcal{T}_{n, \tau_{m}}\right\}$. The C-T design is described by the relationship of the pilot vectors at the second symbol to those at the first symbol. For CDM-F, the pilot vectors at the second symbol are just $\sqrt{-1}$ times the corresponding pilot vectors at the first symbol. For FDM, we can have several approaches such as: (i) the pilot vectors at the second symbol are $\sqrt{-1}$ times those at the first symbol, (ii) the pilot tones with indexes less than $N / 2$ remain the same over two symbols, while the remaining pilots change polarities across the two symbols, or (iii) for each antenna pair with mirror index sets $\mathcal{T}_{n, \tau_{m}}$ and $\mathcal{T}_{n, \tau_{\bar{m}}}$, the antenna using $\mathcal{T}_{n, \tau_{m}}$ transmits the same pilots on both symbols, while the other antenna changes polarities of pilots across the two symbols.

\section{Simulation Results and Discussions}

\section{A. Parameter Setting}

System parameters in the simulation are $N_{\mathrm{Tx}}=2, N_{\mathrm{Rx}}=$ 2, $N=128,6$ left and 6 right null guard tones, 16-QAM, and a 4-tap Rayleigh fading channel with an exponential power delay profile ( $3 \mathrm{~dB}$ per tap decay factor). The frequency-independent $\mathrm{I} / \mathrm{Q}$ imbalances are set to $\alpha=\frac{a_{t}^{I}}{a_{t}^{Q}}=\frac{a_{r}^{I}}{a_{r}^{Q}}=1.09648$, and $\Delta \theta=\theta_{t}^{I}-\theta_{t}^{Q}=\theta_{r}^{I}-\theta_{r}^{Q}=3^{\circ}$. The frequencydependent I/Q imbalances are modeled by 3-tap filters with discrete-time impulse responses of $[0.01,0.9999,0.01]$ and $[0.015,0.9998,0.01]$ for the transmit I and Q branches, and $[0.012,0.9997,0.018]$, and $[0.01,0.9997,0.02]$ for the receive I and Q branches. For performance comparison, the design from [16] as MIMO Reference 1 and a design from [2] as MIMO Reference 2. In all methods, the maximum likelihood detection is applied. For a fair comparison, total pilot energies of all methods are set the same.

\section{B. Estimation MSE, BER, and Overhead Comparison}

Fig. 2 presents the channel estimation MSE of the direct and mirror channel. As the MIMO Reference 1 design is suboptimal due to null guard tones, its performance is marginally worse than the proposed designs. As MIMO Reference 2 does not consider I/Q imbalance, it fails when I/Q imbalance is unnegligible. The theoretical MSE in (20) gives an exact match 
TABLE I

Pilot OVERHEAD COMPARISON

\begin{tabular}{||c|c|}
\hline Design & Overhead (\# of Tones) \\
\hline [CDM-F; Null] & $2 N_{\mathrm{Tx}} L_{0}$ \\
\hline [FDM; Null] & $2 N_{\mathrm{Tx}} L_{0}$ \\
\hline [CDM-F or FDM; C-T] & $2 N_{\mathrm{Tx}} L_{0}$ \\
\hline Design from [16] & $2 N_{\mathrm{Tx}} N$ \\
\hline
\end{tabular}

to the simulation results. In Fig. 3, the (uncoded) BER performance of different pilot designs are compared for the MIMO system. The performance of MIMO Ref. 2 is left off as the poor channel estimation performance leads to complete loss of data. Similar conclusions for the MSE performance apply for the BER performance. The pilot overhead comparison is provided in Table I which shows a clear advantage of the proposed designs.

\section{CONCLUSIONS}

I/Q imbalance introduces mirror tone interferences in OFDM systems and imposes additional conditions for optimal pilot designs. Optimal pilot designs developed without I/Q imbalance may experience a substantial performance degradation under I/Q imbalance. The insertion of null guard tones in practical OFDM systems also affects the performance of existing pilot designs developed in the presence of I/Q imbalance. We have presented practically-optimal pilot design criteria for equivalent channel estimation in MIMO OFDM systems with frequency-dependent transmit and receive I/Q imbalances, and have developed several pilot designs satisfying these criteria. With the proposed designs, we have illustrated that improvement over the existing designs can be achieved in terms of overhead efficiency, estimation MSE, BER, and general applicability to different transmission formats (such as preamble-based or pilot-data-multiplexed setup, with or without null guard tones).

\section{REFERENCES}

[1] I. Barhumi, G. Leus, and M. Moonen, "Optimal training design for MIMO OFDM systems in mobile wireless channels," IEEE Trans. Signal Processing, vol. 51, no. 6, pp. 1615-1624, Jun. 2003.

[2] H. Minn and N. Al-Dhahir, "Optimal training signals for MIMO OFDM channel estimation," IEEE Trans. Wireless Commun., vol. 5, no. 5, pp. $1158-1168$, May 2006.

[3] C. L. Liu, "Impacts of I/Q imbalance on QPSK-OFDM-QAM detection," IEEE Trans. Consum. Electron., vol. 44, no. 3, pp. 984-989, Aug. 1998.

[4] M. Valkama, M. Renfors, and V. Koivunen, "Compensation of frequency-selective I/Q imbalances in wideband receivers: models and algorithms," IEEE SPAWC, Mar. 2001, pp. 42-45.

[5] J. Tubbax, B. Come, L. Van der Perre, S. Donnay, M. Engels, M. Moonen, and H. De Man, "Joint compensation of IQ imbalance and frequency offset in OFDM systems," Proc. Radio and Wireless Conference RAWCON, 2003, pp. 39-42.

[6] A. Tarighat and A. H. Sayed, "Joint compensation of transmitter and receiver impairments in OFDM systems," IEEE Trans. Wireless Commun., vol. 6, no. 1, pp. 240-247, Jan. 2007.

[7] D. Tandur and M. Moonen, "Joint adaptive compensation of transmitter and receiver IQ imbalance under carrier frequency offset in OFDMbased systems," IEEE Trans. Signal Proc., 2007, vol. 5, pp. 5246-5252.

[8] W. Kirkland and K. Teo, "I/Q distortion correction for OFDM direct conversion receiver," Electronics Letters, 2003, vol. 39, pp. 131-133
[9] L. Brotje, S. Vogeler, K-D. Kammeyer, "Estimation and correction of transmitter-caused I/Q imbalance in OFDM systems, Proc. 7th Intl. OFDM Workshop, Sept. 2002, pp. 178-182.

[10] Y. Egashira, Y. Tanabe, and K. Sato, "A novel IQ imbalance compensation method with pilot-signals for OFDM system," IEEE VTC Fall, 2006, pp. $1-5$.

[11] A. Tarighat, R. Bagheri, and A. Sayed, "Compensation schemes and performance analysis of IQ imbalances in OFDM receivers," IEEE Trans. Signal Processing, 2005, vol. 53, pp. 3257-3268.

[12] R. Chrabieh and S. Soliman, "IQ Imbalance mitigation via unbiased training sequences," Proc. IEEE GLOBECOM, 2007, pp. 4280-4285.

[13] E. Lopez-Estraviz, S. De Rore, F. Horlin, and A. Bourdoux, "Pilot design for joint channel and frequency-dependent transmit/receive IQ imbalance estimation and compensation in OFDM-based transceivers," IEEE ICC 2007, pp. 4861-4866.

[14] H. Minn and D. Munoz, "Pilot Designs for Channel Estimation of OFDM Systems with Frequency-Dependent I/Q Imbalances," accepted in IEEE WCNC, Apr. 2009.

[15] T. Schenk, P. Smulders, and E. Fledderus, "Estimation and compensation of TX and RX IQ imbalance in OFDM-based MIMO systems," Proc. IEEE Radio and Wireless Symposium, 2006, pp. 215-218.

[16] T. Schenk, P. Smulders, and E. Fledderus, "Estimation and compensation of frequency selective TX/RX IQ imbalance in MIMO OFDM systems," IEEE ICC 2006, vol. 1, pp. 251-256.

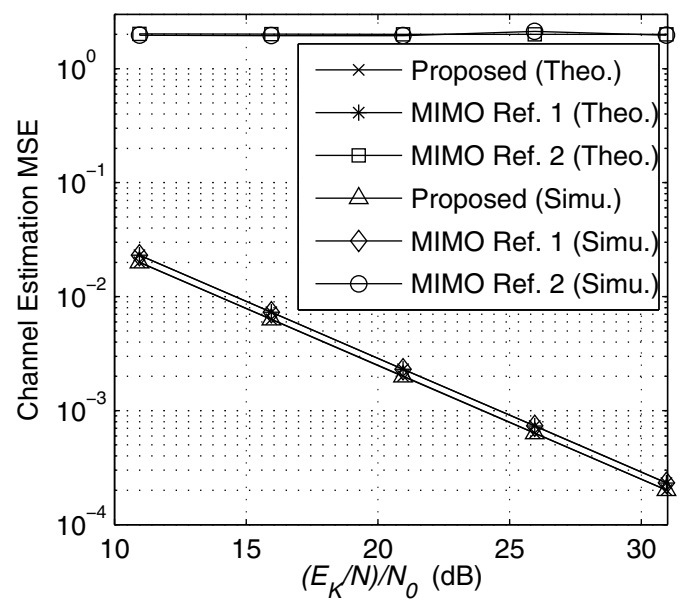

Fig. 2. Channel estimation MSE comparison of different pilot designs in $2 \times 2$ MIMO OFDM system

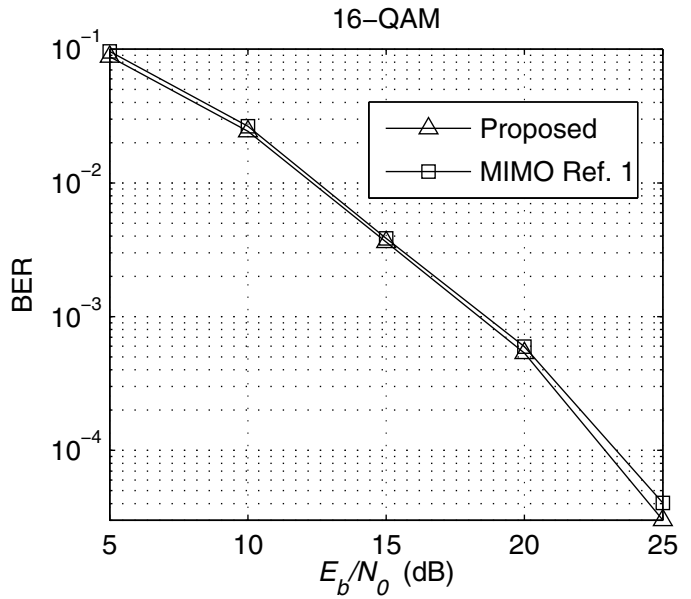

Fig. 3. BER comparison of different pilot designs for 16-QAM in $2 \times 2$ MIMO OFDM system 